\title{
Phase diagram of vertically shaken granular matter
}

\author{
Peter Eshuis \\ Physics of Fluids, University of Twente, P.O. Box 217, 7500 AE Enschede, The Netherlands
}

Ko van der Weele

Department of Mathematics, Division of Applied Analysis, University of Patras, 26500 Patras, Greece

Devaraj van der Meer, Robert Bos, and Detlef Lohse

Physics of Fluids, University of Twente, P.O. Box 217, 7500 AE Enschede, The Netherlands

(Received 14 May 2007; accepted 22 October 2007; published online 6 December 2007)

\begin{abstract}
A shallow, vertically shaken granular bed in a quasi-two-dimensional container is explored experimentally yielding a wider variety of phenomena than in any previous study: (1) bouncing bed, (2) undulations, (3) granular Leidenfrost effect, (4) convection rolls, and (5) granular gas. These phenomena and the transitions among them are characterized by dimensionless control parameters and combined in a full experimental phase diagram. (C) 2007 American Institute of Physics.
\end{abstract}

[DOI: $10.1063 / 1.2815745$ ]

\section{INTRODUCTION}

Vertically shaken granular matter exhibits a wealth of fluidlike phenomena: Undulations ${ }^{1-3}$ and other wave patterns ${ }^{4,5}$ (comparable to Faraday waves in an ordinary liquid $^{6,7}$ ), the granular Leidenfrost effect ${ }^{8}$ (being the granular version of the synonymous effect of a water drop hovering over a hot plate ${ }^{9}$ ), and convection rolls reminiscent of those found in a fluid heated from below beyond the RayleighBénard instability. ${ }^{10,11}$ However, while in normal fluids and gases these phenomena are fully understood, this is much less the case for their granular counterparts. One of the major challenges in granular research today is to achieve a hydrodynamiclike description of these effects, and although such a description has been given successfully for some isolated cases, we are still far from an overall theory.

An indispensable step towards any such theory, and an important indication of the physical mechanisms at work, is the determination of the dimensionless control parameters that govern the phenomena. Here we present an experimental study of a vibrated bed of glass beads in which we do exactly this: For each observed effect (and the phase transitions between them) we identify the relevant control parameters. The paper culminates in the construction of an experimental phase diagram in which all observed phenomena are combined.

Our experimental setup (Fig. 1) consists of a quasi-twodimensional (quasi-2-D) Perspex ${ }^{\circledR}$ container of dimensions $L \times D \times H=101 \times 5 \times 150 \mathrm{~mm}$ (with $L$ the container length, $D$ the depth, and $H$ the height), partially filled with glass beads of diameter $d=1.0 \mathrm{~mm}$, density $\rho=2600 \mathrm{~kg} / \mathrm{m}^{3}$, and coefficient of normal restitution $e \approx 0.95$. The setup is mounted on a sinusoidally vibrating shaker with tunable frequency $f$ and amplitude $a$. Most of the experiments presented in this paper are performed by upsweep experiments in which the frequency is increased linearly at $75 \mathrm{~Hz} / \mathrm{min}$. These experiments are recorded with a high-speed camera capturing 2000 frames per run; adequate recording times (4 to $16 \mathrm{~s}$ ) are obtained by adjusting the frame rate.
The natural dimensionless control parameters to analyze the experiments are (i) the shaking parameter $a^{2} \omega^{2} / g \ell$ (with $\omega=2 \pi f$ and $g=9.81 \mathrm{~m} / \mathrm{s}^{2}$ ), being the ratio of the kinetic energy inserted into the system by the vibrating bottom and the potential energy associated with a typical displacement of the particles $\ell$; (ii) the number of bead layers $F$; (iii) the inelasticity parameter $\varepsilon=\left(1-e^{2}\right)$; and (iv) the aspect ratio $L / h_{0}$, where $h_{0}$ denotes the bed height at rest. The parameter $\varepsilon$ is taken to be constant in this paper, since we ignore the velocity dependence and use the same beads throughout. The aspect ratio varies by changing the bed height $h_{0}$ (i.e., the number of layers $F$ ) but remains large in all experiments; i.e., $L / h_{0} \gg 1$. We will systematically vary the first two dimensionless parameters, by changing the amplitude $a$, the frequency $f$, and the number of layers $F$.

The most intriguing of the four parameters above is the first one, the shaking parameter, since the typical displacement of the particles $\ell$ is influenced in a nontrivial way by the vibration intensity and the number of particle layers. For mild fluidization, the displacement of the particles is determined by the amplitude of shaking $a$, since the bed closely follows the motion of the bottom. The energy ratio in this case becomes identical to the well-known dimensionless shaking acceleration

$$
\Gamma=\frac{a \omega^{2}}{g} .
$$

For strong fluidization, the particles no longer follow the bottom, so (instead of $a$ ) some intrinsic length scale needs to be taken for $\ell$, such as the particle diameter $d$. This leads to the dimensionless shaking strength $S$ (see Refs. 8 and 12)

$$
S=\frac{a^{2} \omega^{2}}{g d} .
$$

At intermediate fluidization, we will encounter phenomena in which there is a competition of length scales. In this region, the transitions are affected by changing one of the competing length scales, meaning that the choice of the appro- 


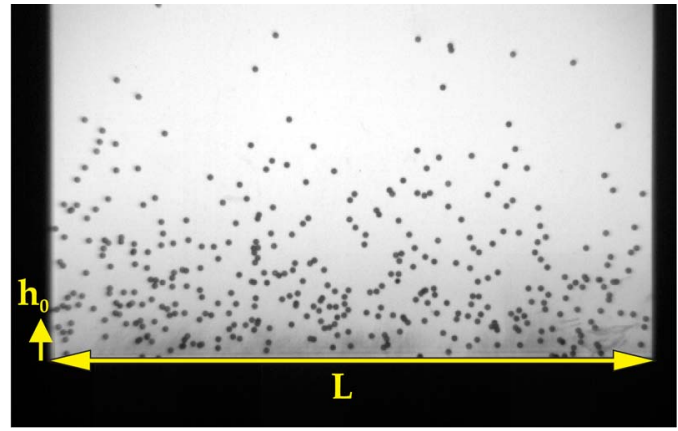

FIG. 1. The experimental setup in which glass beads of diameter $d$ $=1.0 \mathrm{~mm}$ are vibrofluidized. The length of the container is $L=101 \mathrm{~mm}$; the bed height at rest $\left(h_{0}\right)$ is varied in our experiments such that the aspect ratio $L / h_{0}$ always remains large. The container depth is only five particle diameters, making the setup quasi-two-dimensional.

priate shaking parameter is not a priori clear. This will become an issue in particular for the transition from undulations to the granular Leidenfrost effect described in Sec. IV.

In the following sections, the various phenomena observed in our system are discussed one by one, in the order in which they appear as the fluidization is increased: bouncing bed (Sec. II), undulations (Sec. III), granular Leidenfrost effect (Sec. IV), convection rolls (Sec. V), and granular gas (Sec. VI). Finally, in Sec. VII, all five phenomena will be combined in a phase diagram of the relevant shaking parameter versus the number of layers.

\section{BOUNCING BED}

For shaking accelerations $\Gamma \leq 1$ (and even for $\Gamma$ slightly above 1) the granular bed behaves as a solid, comoving with the vibrating bottom and never detaching from it. In order to detach, the bottom must at some point during the cycle have a downward acceleration that overcomes gravity (as for a single bouncing ball ${ }^{13,14}$ ) plus the friction between the bed and the walls of the container. These walls carry a considerable portion of the bed weight, as described in the RayleighJanssen model ${ }^{15,16}$ by the detachment condition for the dimensionless shaking acceleration:

$$
\Gamma_{\text {detach }}=2-e^{-\chi}, \quad \text { with } \chi=K \mu_{s} \xi .
$$

Here, $\chi$ is the decompaction parameter, which is defined by the coefficient of redirection toward the wall $K$, the static friction coefficient for Perspex ${ }^{\circledR} \mu_{s}=0.8$ and the ratio of the contact area over the cross-sectional area $\xi$,

$$
\xi=\frac{P h_{0}}{A}=\frac{2(D+L) h_{0}}{D L} \quad \rightarrow \quad \xi=\frac{2 h_{0}}{D} \quad(D \ll L),
$$

where $P$ is the perimeter, $h_{0}$ the bed height at rest, and $A$ the cross-sectional area of the container. Once the detachment condition of Eq. (3) is fulfilled, the bed bounces in a similar way as a single particle would do: We call this a bouncing bed (see Fig. 2).

The value of $\Gamma$ at which the transition from solid to bouncing bed occurs in experiment has been determined by gradually increasing the frequency $f$ (for three fixed shaking amplitudes: $a=2.0,3.0$, and $4.0 \mathrm{~mm}$ ). The onset value grows
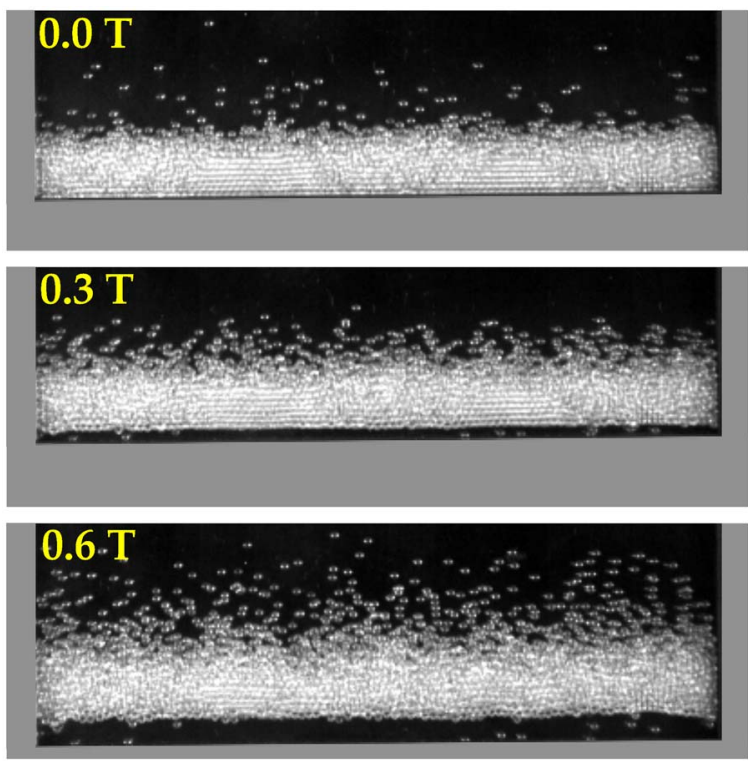

FIG. 2. Time-series of a bouncing bed for $F=8.1$ layers of $d=1.0 \mathrm{~mm}$ glass beads at shaking acceleration $\Gamma=2.3(a=4.0 \mathrm{~mm}, f=12.0 \mathrm{~Hz})$. The phase of the sinusoidally vibrating bottom is indicated in each snapshot, where $T$ is the period of shaking $\left[y_{\text {bottom }}(t)=a \sin (2 \pi t / T)\right]$. The friction between the particles and the container walls causes the downward curvature of the bed close to the sidewalls that is visible in the lower snapshot (enhanced online).

with the number of layers $F$, as shown in Fig. 3. The reason for this is the larger contact area with the front- and sidewalls (larger $\xi$ ) causing a proportionally higher frictional force, leading to a higher value of $\Gamma_{\text {detach }}$, as described by Eq. (3). To compare the model with the experiments we have to take into account that the forces in our quasi-2-D setup $(D \ll L)$ are redirected weaker in comparison with the threedimensional situation of the Rayleigh-Janssen model. Thus, the redirection coefficient $K$ is expected to be smaller than the value for a compact triangular packing $(K=0.58)$. This is indeed found, the best fit through the experimental data of Fig. 3 is achieved for $K=0.15$.

Figure 3 indicates that for the current transition (which occurs at mild fluidization) $\Gamma$ is a good dimensionless parameter, as explained in Sec. I. It is not ideal, as exemplified by

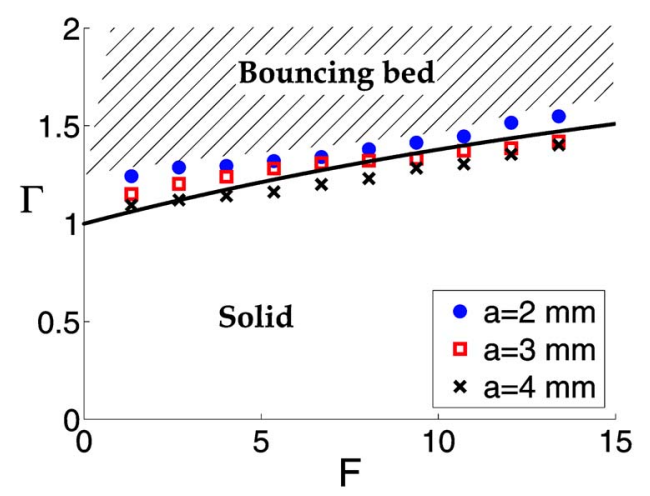

FIG. 3. (Color online) The transition from solid behavior to bouncing bed is governed by the shaking parameter $\Gamma$. The critical value (here determined for three fixed amplitudes: $a=2.0,3.0,4.0 \mathrm{~mm}$ ) increases with the number of particle layers $F$ following the Rayleigh-Janssen model (solid line) with the redirection coefficient $(K=0.15)$ adapted to our quasi-2-D setup. 

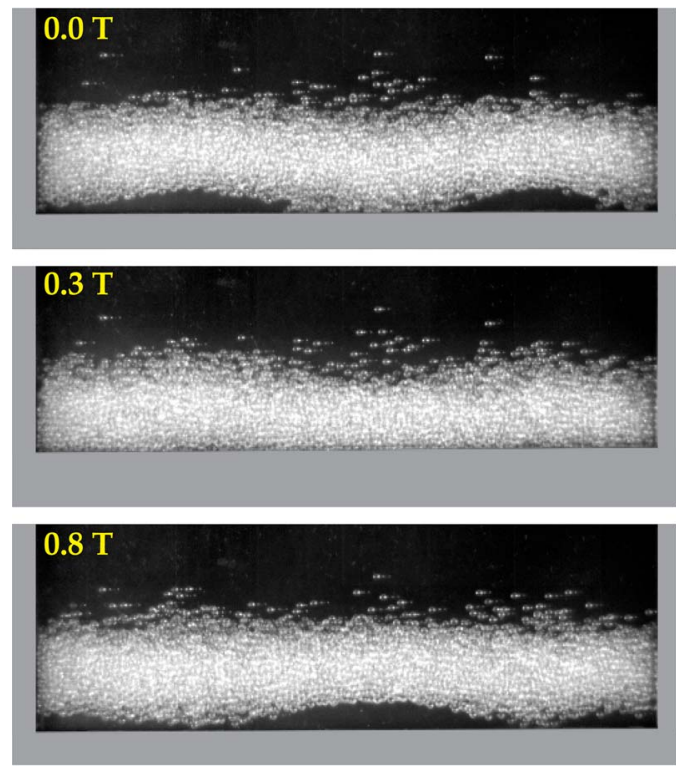

FIG. 4. One complete standing-wave cycle of the $n=4$ undulation mode for $F=9.4$ particle layers at $\Gamma=12(a=2.0 \mathrm{~mm}, f=39.3 \mathrm{~Hz})$. The undulation cycle takes $2 / f$; i.e., twice the period of shaking (enhanced online).

the fact that the onset values do not exactly coincide for the different amplitudes of shaking, but for a different choice of the shaking parameter $(S)$, the onset values differ much more.

\section{UNDULATIONS}

Starting from a bouncing bed and increasing the shaking frequency $f$, three different phenomena are observed: (a) For $F \leqslant 3$ layers, the bed is vaporized and becomes a granular gas (Sec. VI); (b) for $3<F \leqslant 6$, convection rolls form (Sec. $\mathrm{V}$ ); and (c) for $F>6$ layers, the bed develops standing waves oscillating at twice the period of shaking (known as "undulations," "arches," "ripples," or " $f / 2$-waves"1,3-5,17), and these will be covered in this section.

In the undulations regime, the granular bed shows standing wave patterns similar to a vibrating string, as shown in Fig. 4. The container (length $L$ ) accommodates an integer number $n$ of half-wavelengths of the granular string,

$$
L=n \frac{\lambda}{2}, \quad n=1,2,3, \ldots,
$$

where $\lambda$ is the length of one arch in the undulation pattern. This $\lambda$ represents a new length scale in the system besides the shaking amplitude $a$ and the particle diameter $d$. Unlike these previous length scales, $\lambda$ is connected to the elastic properties of the particles, which play an important role in the undulations.

We observe that each collision with the bottom causes a shock wave through the bed at a roughly constant speed $v$. This sends compaction waves along the arch, starting out from the lower parts and meeting in the center. At this point the waves bring each other to a halt and the center falls down to the bottom. (At the same time, the previous lower parts are now elevated.) This occurs after one shaking period and the collision with the bottom generates new shock waves, repeat- ing the series of events. In our experiments the undulation modes are always perpendicular to the sidewalls; i.e., they show either a minimum or a maximum there. This same boundary condition was also found by Sano. ${ }^{3}$ We propose the following physical reason: Whenever the bed does not move perpendicularly to the wall, the particles will bounce off the sidewall instead of being halted by it, and as a result the undulation mode is adjusted or shifted until it is perpendicular to the wall.

Since it takes precisely two shaking periods to complete one full oscillation of the undulation pattern (meaning that the minima and maxima exchange positions every vibration cycle), the successive undulation modes appear with increasing steps of half a wavelength.

Generally, the first undulation to be formed is the $n=1$ mode, and for increasing shaking intensity the higher modes depicted in Fig. 5 successively appear. They are triggered by the horizontal dilatancy the bed experiences when it collides with the vibrating bottom: ${ }^{3}$ the string of particles along the bottom dilates and beyond a certain threshold, buckling will occur, which forces the particles into an arch. Using this physical picture, $\mathrm{Sano}^{3}$ was able to derive a theoretical form of the undulation modes, which agrees with the form of the experimental ones in Fig. 5. Let $s$ denote the position along the length of the layer, following the contour of the undulation, and $\theta(s)$ the angle the bed makes (at position $s$ ) with the horizontal $x$ axis. This angle is governed by ${ }^{3}$

$$
\frac{d^{2} \theta}{d s^{2}}=-\alpha^{2} \sin \theta
$$

Here, $\alpha=\sqrt{F / \tilde{E} I}$, with $F$ the reaction force from the side walls upon both ends of the bed, $\widetilde{E}$ the effective Young's modulus of the bed, and $I$ its moment of inertia.

Equation (6) is the well-known pendulum equation with $s$ instead of the time $t$. It can be solved analytically in terms of the Jacobi elliptic functions, ${ }^{3}$ but for our purposes it is sufficient to consider the small angle approximation $\sin \theta$ $\approx \theta$, which simplifies the problem to that of a harmonic oscillator. Inserting the boundary conditions $\theta(0)=0$ and $\theta(L)$ $=0$ (the bed is horizontal at both ends, as discussed above), this yields the following solution:

$$
\theta(s)=\theta_{\max } \sin \left(\frac{n \pi s}{L}\right)
$$

with $\theta_{\max }$ denoting the maximal angle with the horizontal $x$ axis (which is an increasing function of the mode number $n$ ). In the small angle approximation, the horizontal distance $x$ is equal to the measured length along the undulation layer $(x$ $\approx s$ ), so the shape of the undulation modes can simply be calculated by integrating Eq. (7) over $x$ :

$$
y(x)=\theta_{\max } \frac{L}{n \pi}\left[1-\cos \left(\frac{n \pi x}{L}\right)\right], \quad n=1,2,3, \ldots .
$$

The profiles generated by Eq. (8) match the experimental modes of Fig. 5 very well.

In our experiments, we first focused on the transition from the bouncing bed behavior to the $n=1$ mode. In Fig. 6 this transition is shown in the $(\Gamma, F)$-phase diagram for three 

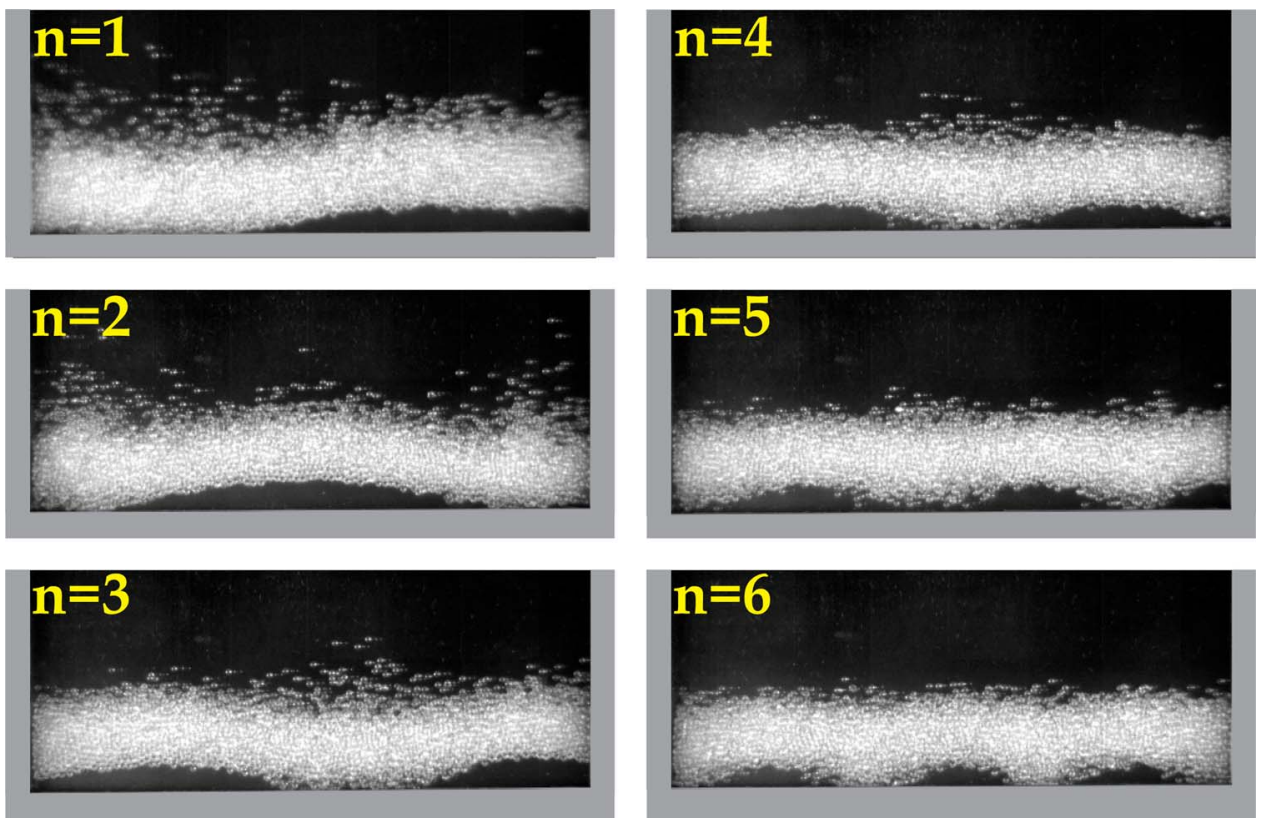

FIG. 5. Six successive undulation modes, for $F=9.4$ layers and $a$ $=2.0 \mathrm{~mm}$, at shaking frequencies $f$ $=29.0,32.6,38.2,39.3,46.1,50.2 \mathrm{~Hz}$. The mode number $n$ (the number of half-wavelengths fitting the container length $L$ ) increases with the shaking intensity. fixed amplitudes of shaking: $a=2.0,3.0$, and $4.0 \mathrm{~mm}$. We observe that the onset value of $\Gamma$ decreases with growing number of layers $F$. The reason for this is that the necessary horizontal dilation (of the lower layer) upon impact with the bottom is more readily accomplished due to pressure from the extra layers on top.

It is seen in Fig. 6 that the data for the three different shaking amplitudes coincide reasonably well, except at the threshold value of $F=6$ layers. Presumably, at this small value of $F$ the dilation can only become sufficient if the density is locally enhanced by a statistical fluctuation; were the experiment repeated many times, the agreement between the averaged data for various $a$ would be expected to become better. For $F<6$ layers no undulations are found, since the particle density is then definitely too small (even in the presence of fluctuations) to reach the required level of dilation.

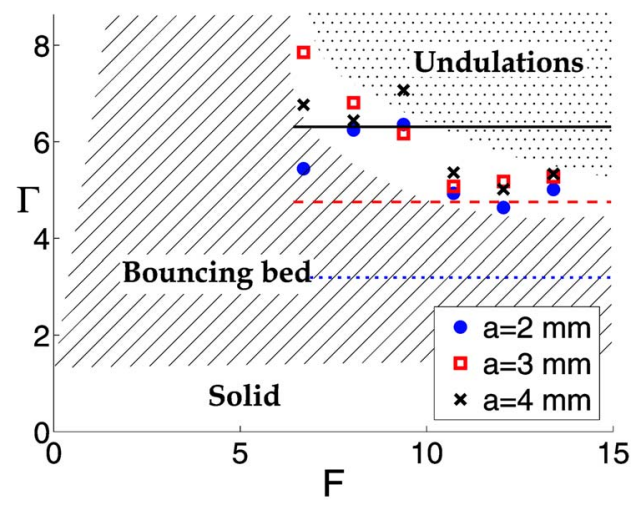

FIG. 6. (Color online) The transition from bouncing bed to undulations in the $(\Gamma, F)$ plane, for three fixed values of the shaking amplitude ( $a$ $=2.0,3.0,4.0 \mathrm{~mm})$. The critical value of the shaking acceleration $\Gamma$ decreases with growing number of particle layers $F$, since the horizontal dilation of the bottom layer (required to trigger undulations, see text) becomes more pronounced as a result of the extra layers on top. The horizontal lines correspond to the onset of undulations predicted by Eq. (9) with $n=1$, where the dotted line (blue online) corresponds to $a=2.0 \mathrm{~mm}$, the dashed line (red online) to $a=3.0 \mathrm{~mm}$ and the solid line (black online) to $a=4.0 \mathrm{~mm}$.
The undulation regime lies in the area of mild fluidization, and Fig. 6 shows that the dimensionless shaking acceleration $\Gamma$ [see Eq. (1)] is indeed the appropriate governing parameter for the undulation phase, in agreement with what has been reported in the literature. Many researchers constructed a phase diagram using $\Gamma=a(2 \pi f)^{2} / g$ in combination with $f^{*}=f \sqrt{h_{0} / g}$, which, however, are not independent of each other. ${ }^{3,5,18,19}$ We use the $(\Gamma, F)$-phase diagram, in which the two control parameters are independent, as was also done by, e.g., Wassgren et al. ${ }^{20}$ and Hsiau and Pan. ${ }^{21}$

Now we come to the higher undulation modes. We have already mentioned the role played by shock waves in the formation of undulations. Such a compaction wave starts out from the lower regions, propagates along the arch, and is halted in the center by its counterpart going in the opposite direction. Hence, these shock waves travel a distance $\frac{1}{2} \lambda$ $=L / n$ in one period of shaking $T=1 / f$; i.e., their speed is given by $v=L f / n$. We know from the experiments that the speed of the shock waves decreases roughly linearly from $v=2 \mathrm{~m} / \mathrm{s}$ for $n=1$, to $v=1 \mathrm{~m} / \mathrm{s}$ for the $n=6$ mode, caused by the lower density inside the granular bed at higher fluidization. Thus, we can estimate the shaking frequency $f_{n}$ at which a certain mode will appear:

$$
f_{n}=\frac{n v(n)}{L} .
$$

Equation (9) predicts the onset of undulations [i.e., the first mode $n=1$, with $v(1)=2 \mathrm{~m} / \mathrm{s}$ ] reasonably well, as shown in Fig. 6. The higher undulation modes observed for shaking amplitude $a=2.0 \mathrm{~mm}$ are displayed in Fig. 7 along with the location of the transitions for the various undulation modes $n$ based on Eq. (9). The location of these transitions is a fair match to the experimental findings, which may be taken as a confirmation of the shock-wave mechanism described above.

As already observed in Fig. 5 and demonstrated in Eq. (9), the mode number $n$ increases for growing $\Gamma$. However, the sequence of modes is seen to be interrupted somewhere 


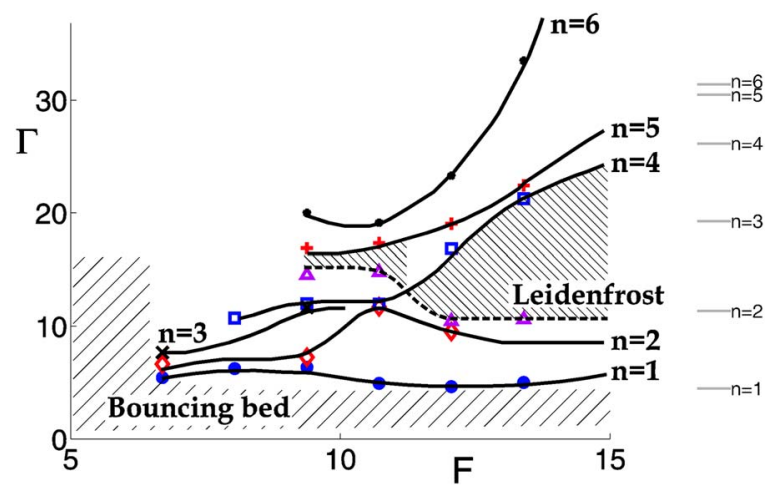

FIG. 7. The onset of the successive undulation modes $n=1,2, \ldots, 6$ at a fixed shaking amplitude $a=2.0 \mathrm{~mm}$. The mode number $n$ increases with the shaking parameter $\Gamma$, but occasionally the undulations give way to the granular Leidenfrost effect (the hatched regions above the dashed curve), where a dense cluster without any arches is floating on a uniformly dilute granular layer. The gray lines on the right indicate the location of the various undulation modes based on Eq. (9) and agree reasonably with the experimental observations.

in the middle: Here the undulation pattern gives way to the granular Leidenfrost state, ${ }^{8}$ in which a cluster of slow particles is floating on top of a dilute layer of fast particles. Normally, this state appears at the end of the undulation regime (see Sec. IV), but when a certain standing wave pattern is energetically unfavorable, the system chooses the Leidenfrost state instead: In Fig. 7 we see that this happens to the $n=3$ undulation, which is completely skipped from the sequence for $F \gtrsim 12$ layers. This can be understood from the fact that the $n=3$ mode has an antinode at the sidewall (i.e., a highly mobile region), whereas the friction with the wall tends to slow down the particles here. This inherent frustration gives rise to the appearance of the granular Leidenfrost effect.

Likewise, the small Leidenfrost region for $9 \leq F \leq 12$ below the onset line of the $n=5$ undulation has to do with a frustrated $n=5$ mode. The frustration is, however, not strong enough to skip the mode entirely as in the $n=3$ case. In our experiments, the intermediate Leidenfrost regions become smaller for larger shaking amplitude $a$. For $a=4.0 \mathrm{~mm}$ they have disappeared altogether from the undulation regime, as will be shown in Sec. VII.

\section{GRANULAR LEIDENFROST EFFECT}

When the shaking frequency is increased beyond a critical level, the highest undulation mode becomes unstable and we enter the granular Leidenfrost regime: ${ }^{8}$ Here a dense cloud of particles is elevated and supported by a dilute gaseous layer of fast beads underneath (see Fig. 8). The bottom layer of the undulations is completely evaporated and forms the gaseous region on which the cluster floats. The phenomenon is analogous to the original Leidenfrost effect in which a water droplet hovers over a hot plate on its own vapor layer, when the temperature of the plate exceeds a critical value. ${ }^{9}$ The vaporized lower part of the drop provides a cushion to hover on, and strongly diminishes the heat contact between the plate and the drop, enabling it to survive for a relatively long time.
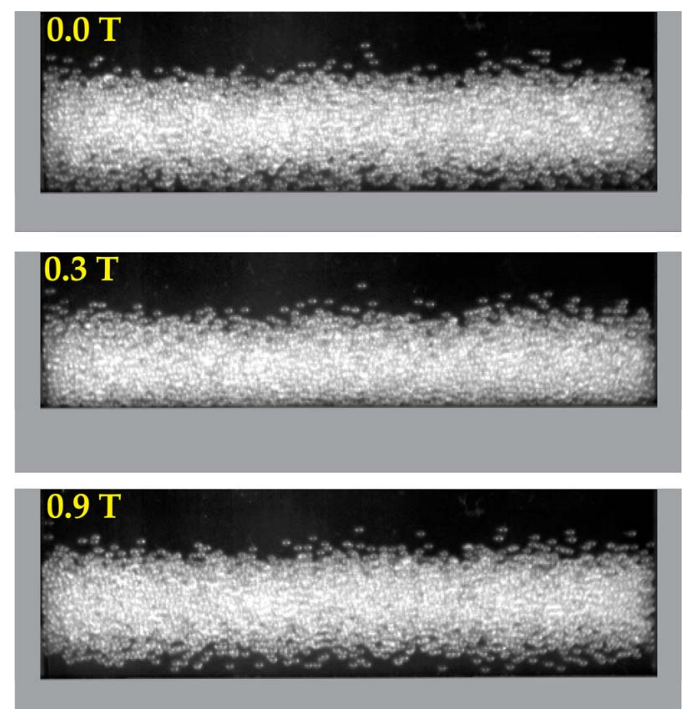

FIG. 8. Snapshots of the granular Leidenfrost effect for $F=8.1$ particle layers shaken at $f=43.0 \mathrm{~Hz}$ and $a=3.0 \mathrm{~mm}$ (corresponding to a dimensionless acceleration $\Gamma=22$ or shaking strength $S=67$ ). A dense cluster is elevated and supported by a dilute layer of fast particles underneath. The cluster never touches the vibrating bottom, which makes this state distinctively different from the bouncing bed or the undulations (enhanced online).

In Fig. 9, the transition from the undulations to the granular Leidenfrost state is shown, both in the $(\Gamma, F)$ and in the $(S, F)$ planes. Despite the fact that we have left the mild fluidization regime behind, $\Gamma$ still appears to be the govern-
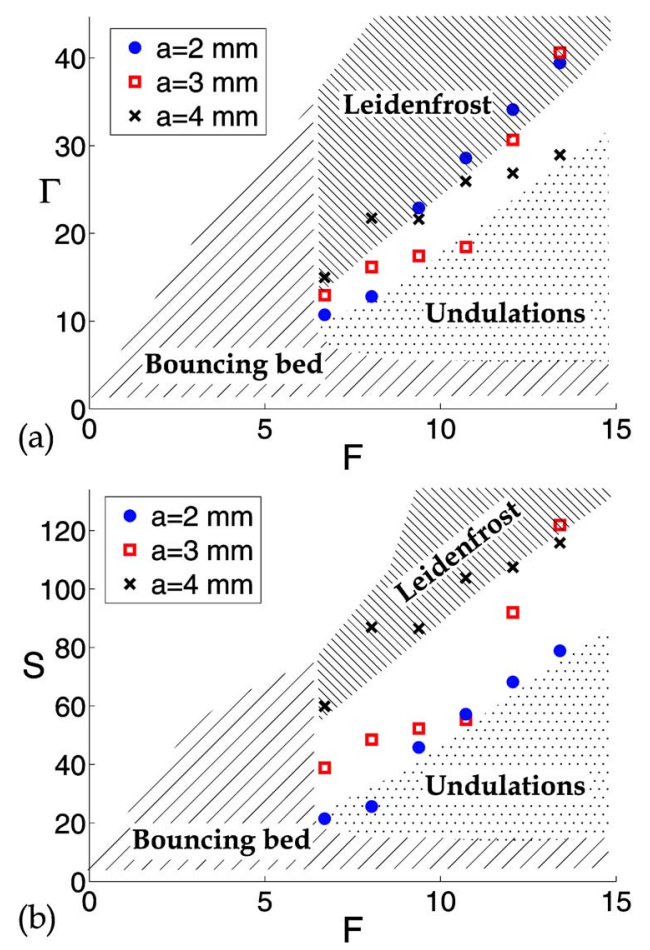

FIG. 9. (Color online) The transition from undulations to the granular Leidenfrost effect for increasing frequency $f$ and fixed amplitude $a$ $=2.0,3.0,4.0 \mathrm{~mm}$ : (a) In the $(\Gamma, F)$ plane, $(\mathrm{b})$ in the $(S, F)$ plane. Since in our experiments the Leidenfrost state always originates from the undulation regime, the same minimum number of layers is needed: $F>6$. The critical values of $\Gamma$ and $S$ increase with $F$, since a higher energy input is required to elevate a larger cluster. 
ing shaking parameter, since the data for the different amplitudes $(a=2.0,3.0,4.0 \mathrm{~mm})$ collapse better on a single curve in the $(\Gamma, F)$ than in the $(S, F)$ plane. In fact, the critical $S$-values in the latter plane show a systematic increase for growing amplitude $a$.

This is in contrast to the observations on the granular Leidenfrost effect in a previous study of smaller aspect ratio, ${ }^{8,22}$ for $d=4.0 \mathrm{~mm}$ glass beads in a 2-D container, where the phase transition was shown to be governed by the dimensionless shaking strength $S$. In that case the Leidenfrost state was reached directly from the solid bouncing bed regime, without the intermediate stage of undulations. Presumably this was due to the much smaller aspect ratio $L / h_{0}$, which was on the order of 1 (against $L / h_{0} \sim 10$ in the present Leidenfrost experiments). ${ }^{23}$ Another important difference was that the depth of the setup was just slightly more than one particle diameter (against five diameters in the present setup), so the motion of the granular bed was much more restricted; indeed, the floating cluster in Ref. 8 showed a distinctly crystalline packing. It may be concluded, as already remarked in Sec. I, that the Leidenfrost effect lies in the regime of intermediate fluidization, where both $\Gamma$ and $S$ are candidates to describe the behavior of the granular bed. The proper choice of the shaking parameter here depends not only on the degree of fluidization, but also on the dimensions of the specific system investigated.

It has been shown that the granular Leidenfrost effect observed in the 2-D container of Ref. 8 is successfully explained by a continuum description based on the hydrodynamic equations. The first one is the equation of state:

$$
p=n T \frac{n_{c}+n}{n_{c}-n}, \quad n_{c}=\frac{2}{\sqrt{3} d^{2}} .
$$

Here, $p$ is the pressure, $n$ the number density with $n_{c}$ the density of a hexagonal close packing, and $T$ the granular temperature. The second equation is the force balance,

$$
\frac{d p}{d y}=-m g n,
$$

where $m$ is the mass of a single particle and $g$ the gravitational acceleration. Finally we have the energy balance,

$$
\frac{d}{d y}\left(\kappa \frac{d T}{d y}\right)=\frac{C_{1}}{\ell} \varepsilon n T^{3 / 2},
$$

in which $\kappa$ is the thermal conductivity, $\ell$ the mean free path, $\varepsilon=\left(1-e^{2}\right)$ the inelasticity parameter, and $C_{1}$ is a constant.

The model described by Eqs. (10)-(12) is closed by three boundary conditions: (1) A prescribed granular temperature at the bottom $T_{0}=$ const, (2) a vanishing energy flux $[\kappa(y) d T / d y=0]$ at the top of the system, and (3) the conservation of particles $\int_{0}^{\infty} n(y) d y=F n_{c} d$. In Ref. 8, this set of equations plus boundary conditions is solved numerically and the resulting density profiles agree quantitatively with the experimental profiles. Thus, the experimental results are successfully captured by the hydrodynamic model.
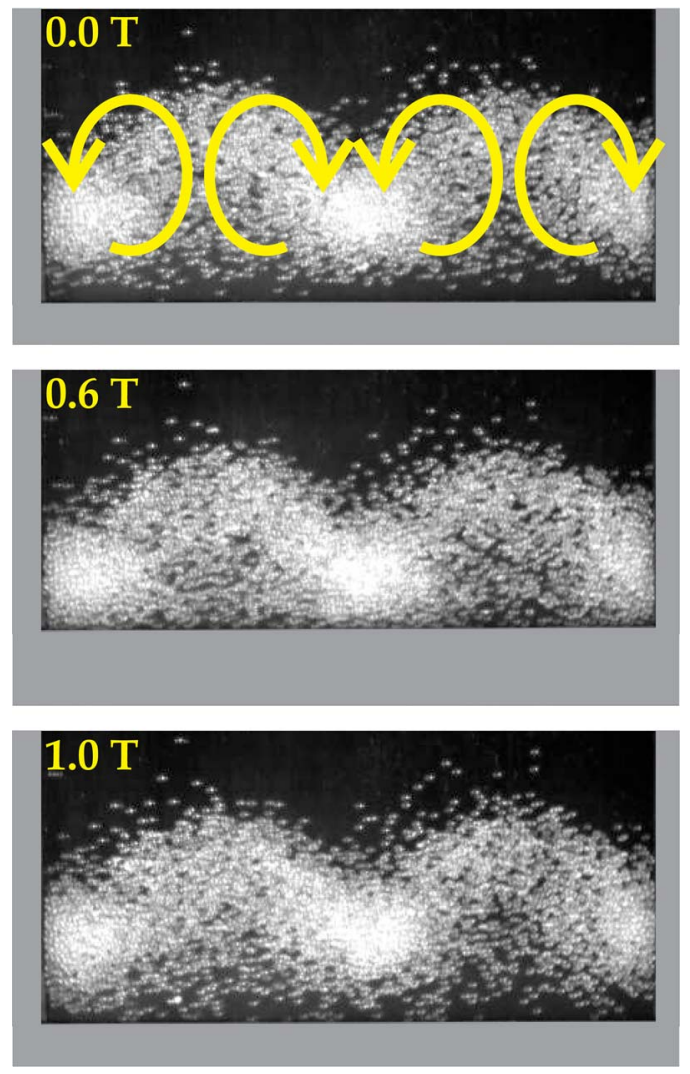

FIG. 10. Granular convection for $F=8.1$ layers at $f=73.0 \mathrm{~Hz}$ and $a$ $=3.0 \mathrm{~mm}$ (dimensionless shaking strength $S=193$ ), showing four counterrotating rolls. The beads move up in the dilute regions (high granular temperature) and are sprayed sideways to the three dense clusters (low granular temperature). In our system two clusters are always located near the sidewalls, which have a relatively low granular temperature due to the extra dissipation (enhanced online).

\section{CONVECTION ROLLS}

In our experiments, granular convection rolls are formed at high fluidization from either (a) the bouncing bed (for 3 $<F \leqslant 6$ layers) or (b) the granular Leidenfrost effect (for $F$ $>6$ ). In both cases the onset of convection is caused by a set of particles in the cluster that are more mobile (higher granular temperature) than the surrounding area, creating an opening in the bed. These particles have picked up an excess of energy from the vibrating bottom (due to a statistical fluctuation) and collectively move upwards, very much like the onset of Rayleigh-Bénard convection in a classical fluid heated from below. ${ }^{10,24}$ This upward motion of the highly mobile beads must be balanced by a downward movement of neighboring particles, leading to the formation of a convection roll.

The downward motion is most easily accomplished at the sidewalls, due to the extra source of dissipation (i.e., the friction with the walls), and for this reason the first convection roll is always initiated near one of the two sidewalls. Within a second, this first roll triggers the formation of rolls throughout the entire length of the container, leading to a fully developed convection pattern as in Fig. 10.

The convection rolls of Fig. 10 are fundamentally different from the rolls reported in the literature: Extensive re- 


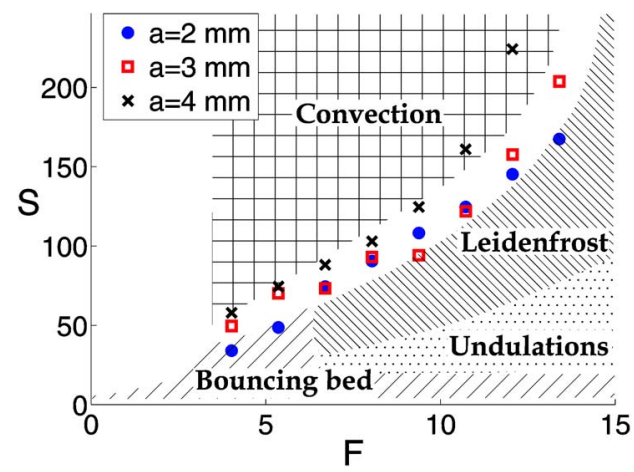

FIG. 11. (Color online) The transition towards granular convection from the bouncing bed $(3<F \leqslant 6)$ and the Leidenfrost state $(F>6)$ in the $(S, F)$ plane, for fixed shaking amplitude $a=2.0,3.0$, and $4.0 \mathrm{~mm}$. Just as for the Leidenfrost transition, the convection sets in at higher values of $S$ as the number of layers $F$ is increased, because a higher dissipation must be overcome for larger bed heights.

search has been done on granular convection experimentally, ${ }^{25-35}$ numerically, ${ }^{36-46}$ and theoretically. ${ }^{47-51}$ All studies deal with a mild fluidization (typically $\Gamma<10$ ) for which the convection is principally boundary driven and with a nearly constant density in the system. The convection observed here, however, occurs at strong fluidization and the rolls show large density differences; i.e., we report of buoyancy-driven convection rather than boundary driven, which is therefore distinctly different. We are aware of only one numerical study, by Paolotti et al. ${ }^{11}$ showing the same kind of rolls with large density gradients, and we here present the first experimental observations. In the numerical model by Paolotti et al. the container walls were taken to be perfectly elastic, leading to convection patterns in which the rolls were either moving up or down along the sidewalls, whereas in our system (with dissipative walls) they always move down.

To theoretically describe this buoyancy-driven convection we have expanded the one-dimensional hydrodynamic model of the granular Leidenfrost effect (see Sec. IV) to a 2-D model, similar to the approach by Khain and Meerson. ${ }^{50}$ The set of equations is linearized around the solution for the granular Leidenfrost state and a stability analysis then yields the point at which the Leidenfrost state gives way to convection rolls. The analysis will be discussed in detail in a future publication.

Figure 11 shows the transition to convection in the $(S, F)$ plane, starting from either the bouncing bed or the Leiden- frost state, which are taken together because the transition dynamics is the same in both cases. This is the first instance in which the data points (acquired for all shaking amplitudes: $a=2.0,3.0$, and $4.0 \mathrm{~mm}$ ) collapse better for the shaking parameter $S$ than for the dimensionless acceleration $\Gamma$, meaning that $S$ is the preferred control parameter for the convection transition.

The onset values of $S$ grow with the number of layers $F$, because for large $F$ more energy input from the vibrating bottom is necessary to break through the larger dissipation in the granular bed and trigger the first convection roll. Related to this, the number of rolls in the convection pattern decreases for growing $F$ : Due to the larger total dissipation, the dense clusters of each roll grow in size. Hence, the convection rolls become wider, meaning that fewer rolls fit into the container.

When, for a given number of layers $F$, the shaking strength $S$ is increased (either via the frequency $f$ or the amplitude $a$ ), the number of rolls in the convection pattern becomes smaller. This is illustrated in Fig. 12: The higher energy input induces expansion of the convection rolls, and the number of rolls decreases stepwise as $S$ is increased. The steps involve two rolls at a time, since the pattern always contains an even number of rolls due to the downward motion imposed by the sidewalls.

\section{GRANULAR GAS}

In this section we briefly discuss the fifth and last phenomenon observed in our system: A granular gas, being a dilute cloud of particles moving randomly throughout the container as in Fig. 13. This state has also been seen in various other experimental systems and is well described by hydrodynamiclike models found in the literature. ${ }^{52,53} \mathrm{In}$ fact, one can use the same continuum description as for the granular Leidenfrost effect (Sec. IV): For a granular gas, the equation of state of Eq. (10) simplifies to the ideal gas law $p$ $=n T$, since the density in a gas is always smaller than the critical number density $\left(n \ll n_{c}\right)$. The force balance of Eq. (11) remains the same for a gas and in the energy balance of Eq. (12) the thermal conductivity $\kappa$ is no longer a function of the height, but a constant. This set of equations is accompanied by boundary conditions and forms a model that accurately describes the experimental observations.

In our setup the gas state is observed only for a small number of layers $(F \leqslant 3)$ and always originates from the bouncing bed regime. At these small $F$, the bed shows ex-
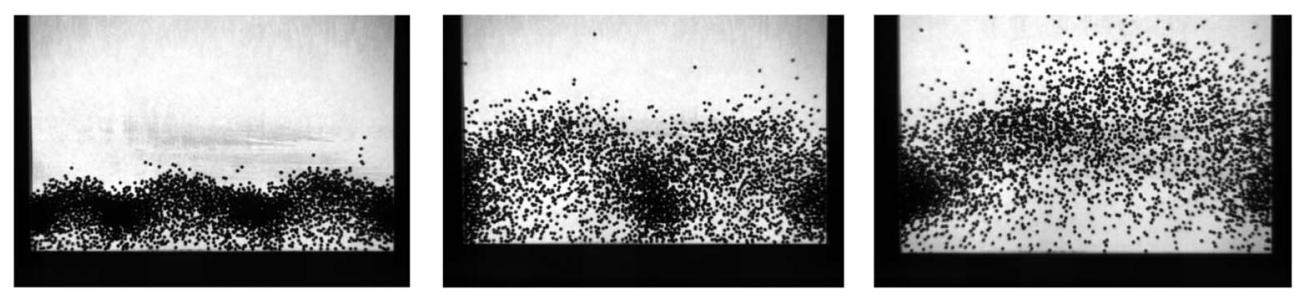

FIG. 12. Convection patterns for $F=6.2$ layers of $1.0 \mathrm{~mm}$ stainless steel beads at three consecutive shaking strengths: $S=58(a=2.0 \mathrm{~mm}, f=60.0 \mathrm{~Hz}), S$ $=130(a=3.0 \mathrm{~mm}, f=60.0 \mathrm{~Hz})$, and $S=202(a=4.0 \mathrm{~mm}, f=56.0 \mathrm{~Hz})$. For increasing $S$, the convection rolls expand; hence, a smaller number of them fits into the container. The steel beads behave qualitatively (but not quantitatively) the same as the glass beads used in the rest of the paper. 

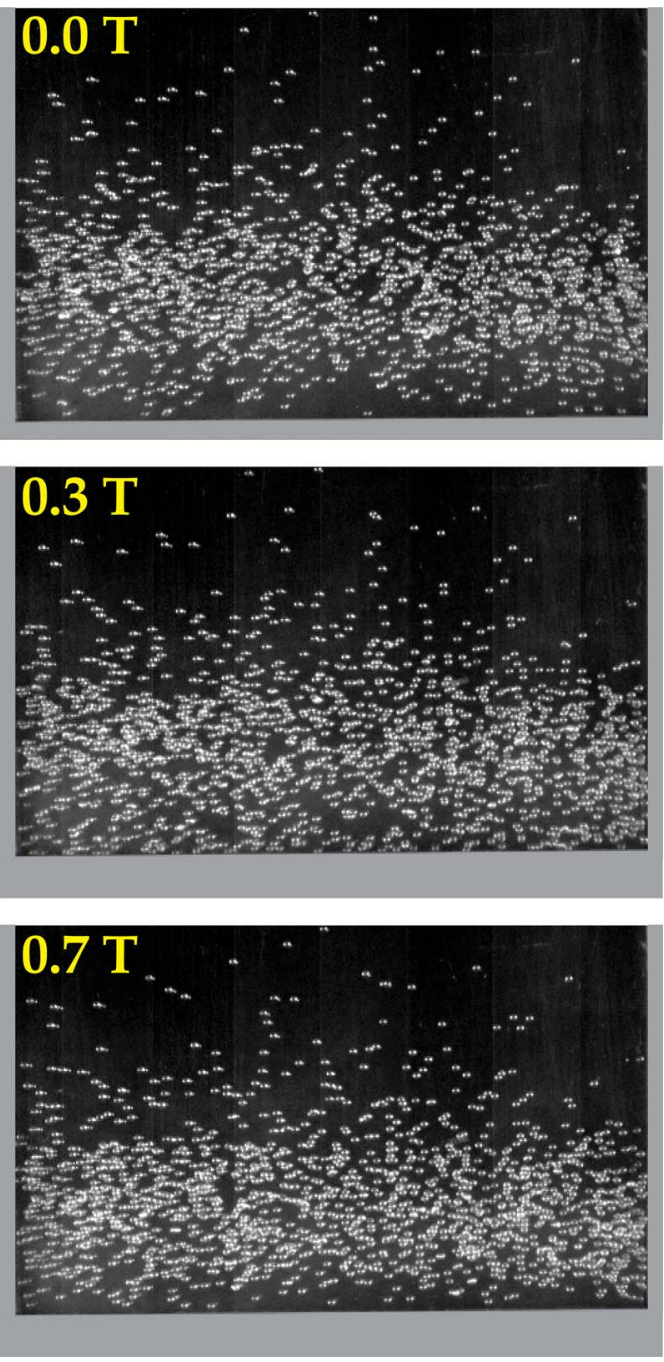

FIG. 13. Granular gas for $F=2.7$ layers at $f=50.0 \mathrm{~Hz}$ and $a=3.0 \mathrm{~mm}(\Gamma$ $=30$ ), which has originated from a bouncing bed by increasing the shaking beyond a critical level [see Fig. 14(b)]. With the vibration power available in our system, granular gases are only observed for $F \leqslant 3$ layers (enhanced online).

pansion and compaction during every vibration cycle due to the low total dissipation. At the critical value of the shaking parameter, the bed expands to such an extent that it evaporates and forms a gas.

The evaporation of the bouncing bed requires more energy as the number of layers $F$ increases. The transition seems to be controlled by the shaking acceleration $\Gamma$ (which also governs the transition from solid to bouncing bed) rather than the shaking strength $S$. However, the data points available are too few $(F \leqslant 3)$ to make this conclusive. The measurements will be presented in the full phase diagram of the next section.

\section{PHASE DIAGRAM}

Finally, all the phenomena and associated transitions described in the previous sections are combined in the phase diagram of Fig. 14. Both shaking parameters $(\Gamma$ and $S)$ are used in this diagram, each of them indicating the respective transitions they were found to govern. The parameter $\Gamma$ is shown along the left vertical axis and the corresponding data points (the critical $\Gamma$ values) are colored red (online). The parameter $S$ is plotted along the right vertical axis and the corresponding experimental data are colored blue (online); this concerns only the " + " signs at the convection transition. ${ }^{54}$ For comparison, the $\Gamma$ axis is kept the same in all three phase diagrams.

Figure 14 contains three separate phase diagrams for the three fixed shaking amplitudes we have used throughout the paper: $a=2.0,3.0$, and $4.0 \mathrm{~mm}$. Most of the phase transitions are hardly affected, with the exception of the various transitions between the undulations and the Leidenfrost state. These transitions lie in the regime of intermediate fluidization, where the system experiences a competition of length scales: the amplitude $a$, the particle diameter $d$, and additionally the wavelength of the undulations $\lambda$. This becomes especially clear in the phase diagram of Fig. 14(a) for $a$ $=2.0 \mathrm{~mm}$ where the competition results in an alternation of states. By increasing $a$, in Figs. 14(b) and 14(c) it becomes the dominant length scale and the alternation vanishes ultimately.

How does Fig. 14 compare with other phase diagrams for shaken granular matter in the literature?

First we discuss the experimental phase diagram by Wassgren et al. ${ }^{20}$ for a bed of $1.28 \mathrm{~mm}$ glass beads at mild fluidization $(\Gamma \leq 10)$. For increasing $\Gamma$, they observe a series of transitions from a solid bed to undulations ("arching") in qualitative agreement with our own experiments at mild shaking. Their series of transitions is interlaced however with several phenomena (Faraday heaping, surface waves) that are not observed in our system. This is presumably due to the larger depth of their container (12.5 particle diameters, versus 5 in our container, which means that their setup deviates considerably from 2-D) and to the fact that their bed height was typically an order of magnitude larger than ours: The lowest aspect ratio $L / h_{0}$ in their experiments was 2 , versus 10 in our system. Hsiau and Pan, ${ }^{21}$ who conducted experiments in a similar setup in the mild fluidization regime, found the same sequence of phenomena as Wassgren et $a l .{ }^{20}$ Indeed, in three dimensions a much wider variety of phenomena is observed than in 2-D systems, both in experiment ${ }^{4,5,55-68}$ and in numerics, ${ }^{69-74}$ e.g., oscillons, heaping, standing wave patterns like squares, stripes, hexagons, spirals, $f / 2$-waves, $f / 4$-waves, as well as phase bubbles and flat surfaces with kinks. We have restricted ourselves to the shaking amplitude range $a=2.0-4.0 \mathrm{~mm}$, and therefore we do not see oscillons, which are only present for large amplitudes in our setup. Heaping is not observed since the role of air on the $d=1 \mathrm{~mm}$ particles is too small to develop heaps. Furthermore, all the above standing wave patterns are surface phenomena and they are simply not observed because of the quasi-2-D nature of our setup.

Secondly, Sunthar and Kumaran ${ }^{43}$ construct a phase diagram (shaking strength versus number of layers) based on event-driven simulations in a 2-D system with an aspect ratio $L / h_{0} \gtrsim 10$, comparable to ours. At low shaking strength, their 

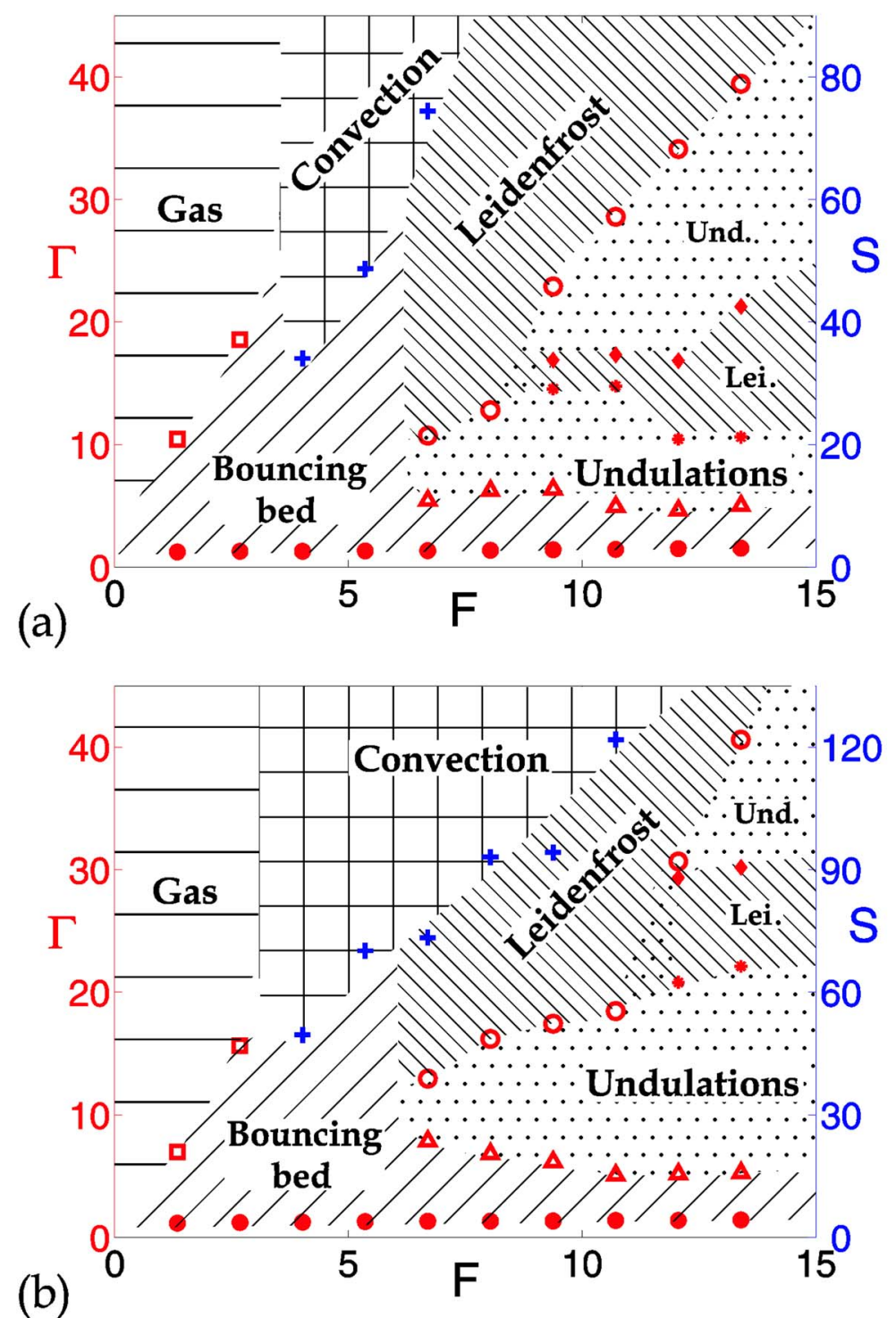

FIG. 14. (Color online) Phase diagram of the shallow
granular bed at three fixed values of the shaking ampli-
tude: (a) $a=2.0 \mathrm{~mm}, \quad$ (b) $a=3.0 \mathrm{~mm}$, and (c) $a$
$=4.0 \mathrm{~mm}$. The five phenomena explored in this paper
are indicated by the different shadings. The onset values
for bouncing bed, undulations, Leidenfrost effect, and
gas are governed by the shaking parameter $\Gamma$ (left ver-
tical axis, red online); the onset of convection is con-
trolled by $S$ (right vertical axis, blue online). The nar-
row region without shading along the horizontal $F$ axis
(below the bouncing bed regime) corresponds to the
solid phase, in which the bed never detaches from the
vibrating bottom (enhanced online).

(b)

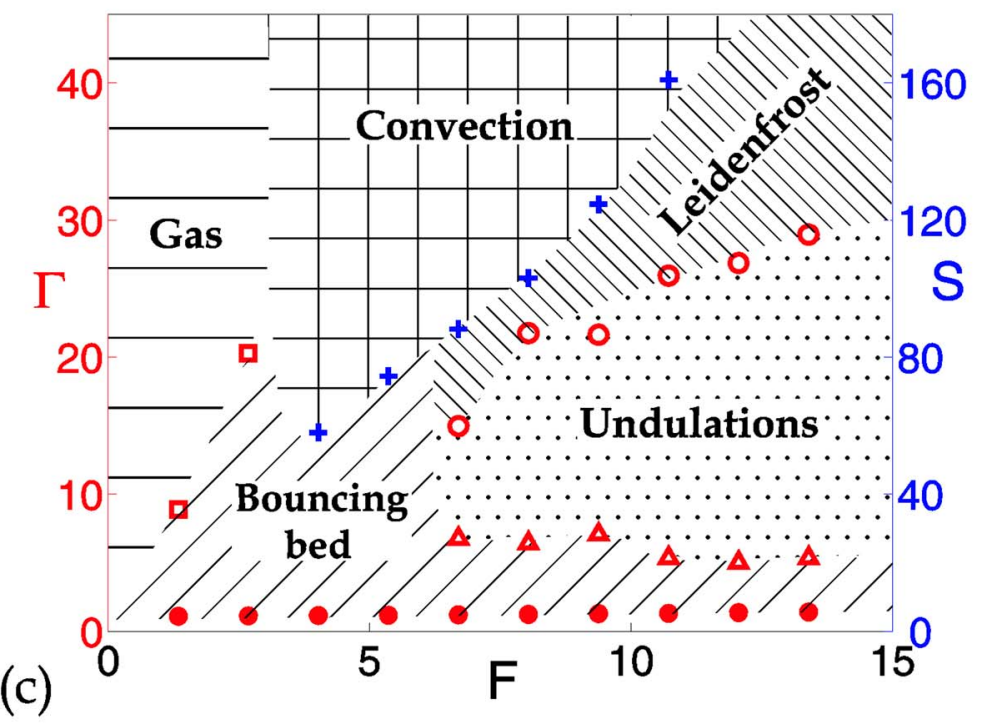

phase diagram shows a region where the bed is "homogeneous," corresponding to the solid and bouncing bed regimes in our diagram. At higher shaking strength, they find a granular gas for $F<5$ and a region of granular convection for $F$
$>5$. The gaseous region compares well with the gas region in Fig. 14. The convection observed by Sunthar and Kumaran, however, occurs at a much milder fluidization than in our system. In contrast to our convection rolls, the density of 
their rolls is almost constant, indicating that the bed behaves more like a fluid than a gas.

Thirdly, Eshuis et al. ${ }^{8}$ construct an experimental phase diagram (supported by a theoretical model) for a bed of $4 \mathrm{~mm}$ glass beads in a 2-D setup. The $(S, F)$ diagram shows a bouncing solid regime for low shaking strength and a gas region for small $F$. Between these two phases, for $S \gtrsim 16$ and $F>8$, the Leidenfrost regime is located. This is qualitatively the same as in Fig. 14, without the regions of undulations and granular convection though. The fact that these latter phenomena were absent is probably due to the much smaller aspect ratio $\left(L / h_{0} \sim 1\right)$ and the much stronger confinement to two dimensions, since the depth of the container was just slightly more than one particle diameter.

Finally, Paolotti et al. ${ }^{11}$ performed a 2-D numerical study of a granular bed with aspect ratio $L / h_{0} \approx 8$, focusing on the transition towards convection. Their convection rolls show arches and distinct density differences similar to those observed in our experiments. Starting from strong fluidization, for a given number of layers, they observe two transitions as the shaking strength is reduced: First, a transition from a nonconvective state (presumably a granular gas) to convection, followed by a transition towards a nonconvective state again, in which the particles remain localized near the bottom. This latter state is not further specified, but most probably corresponds to a bouncing bed. In the phase diagram of Fig. 14 the same sequence is found if one follows a path from the gas regime to the bouncing bed via convection.

The phase diagram of Fig. 14 distinguishes various phase transitions, of which some are phase boundaries and some mark gradual changes. Examples of such a gradual change are the transition from a solid to a bouncing bed, the evaporation of the lower regions of the undulations leading to the floating Leidenfrost cluster, and the expansion of the convection rolls towards a granular gas, which will eventually occur if the shaking strength is increased further. The phenomena observed before and after the transition all display the same symmetry. In contrast, the transitions from a bouncing bed to undulations and the breakthrough of convection rolls starting from the granular Leidenfrost effect show a transition to a state with a different symmetry; they mark the solid-liquid and liquid-gas boundaries, respectively.

A solid-gas phase boundary is also found for small number of layers $(F<6)$, where the bouncing bed expands to either a granular gas or to convection rolls. For a larger number of layers, the solid-liquid phase boundary between the bouncing bed and undulations discriminates between the nonfluidized and fluidized system. When the system is fluidized a hydrodynamic approach is successful, and as we get to a fully fluidized system, the transition from the $\Gamma$-dominated to the $S$-dominated regime is marked by the liquid-gas phase boundary between the Leidenfrost effect and convection.

In conclusion, we have constructed the experimental phase diagram for a vertically shaken shallow granular bed in a quasi-2-D container, identifying the dimensionless control parameters that govern the various transitions in this diagram. In the present work we have concentrated on $\Gamma$ and $S$ (the shaking parameters), and the parameter $F$ (number of particle layers), and we have briefly outlined the current the- oretical models used to describe the various phenomena. From the discussion above, it may be concluded that the aspect ratio is also an important control parameter, determining, e.g., the set of different phenomena that a given system is able to exhibit.

The diagram of Fig. 14 shows the full range of phases that granular matter can display, behaving like a solid, a fluid, or a gas. ${ }^{75-79}$ A determination of the dimensionless parameters that govern the transitions between these phases is a crucial step towards a better understanding of the physics of vertically shaken granular matter.

\section{ACKNOWLEDGMENTS}

The authors thank Stefan Luding for stimulating discussions. This work is part of the research program of FOM, which is financially supported by NWO.

${ }^{1}$ S. Douady, S. Fauve, and C. Laroche, "Subharmonic instabilities and defects in a granular layer under vertical vibrations," Europhys. Lett. 8, 621 (1989).

${ }^{2}$ E. Clément, L. Labous, and L. Vanel, "Granular packing under vibration," Europhys. News 29, 107 (1998).

${ }^{3}$ O. Sano, "Dilatancy, buckling, and undulations on a vertically vibrating granular layer," Phys. Rev. E 72, 051302 (2005).

${ }^{4}$ F. Melo, P. B. Umbanhowar, and H. L. Swinney, "Transition to parametric wave patterns in a vertically oscillated granular layer," Phys. Rev. Lett. 72, 172 (1994).

${ }^{5}$ S. J. Moon, M. D. Shattuck, C. Bizon, D. I. Goldman, J. B. Swift, and H. L. Swinney, "Phase bubbles and spatiotemporal chaos in granular patterns," Phys. Rev. E 65, 011301 (2001).

${ }^{6} \mathrm{M}$. Faraday, "On a peculiar class of acoustical figures; and on certain forms assumed by groups of particles upon vibrating elastic surfaces," Philos. Trans. R. Soc. London 52, 299 (1831).

${ }^{7}$ M. C. Cross and P. C. Hohenberg, "Pattern formation outside of equilibrium,” Rev. Mod. Phys. 65, 851 (1993).

${ }^{8}$ P. Eshuis, K. van der Weele, D. van der Meer, and D. Lohse, "Granular Leidenfrost effect: Experiment and theory of floating particle clusters," Phys. Rev. Lett. 95, 258001 (2005)

${ }^{9}$ J. G. Leidenfrost, "De aquae communis nonnullis qualitatibus tractatus," Int. J. Heat Mass Transfer 9, 1153 (1966).

${ }^{10}$ S. Chandrasekhar, Hydrodynamic and Hydromagnetic Stability (Oxford University Press, Oxford, England, 1961).

${ }^{11}$ D. Paolotti, A. Barrat, U. M. B. Marconi, and A. Puglisi, "Thermal convection in monodisperse and bidisperse granular gases: A simulations study," Phys. Rev. E 69, 061304 (2004).

${ }^{12}$ H. K. Pak and R. P. Behringer, "Surface waves in vertically vibrated granular materials," Phys. Rev. Lett. 71, 1832 (1993).

${ }^{13} \mathrm{P}$. J. Holmes, "The dynamics of repeated impacts with a sinusoidally vibrating table," J. Sound Vib. 84, 173 (1982).

${ }^{14}$ A. Mehta and J. M. Luck, "Novel temporal behavior of a nonlinear dynamical system: The completely inelastic bouncing ball," Phys. Rev. Lett. 65, 393 (1990).

${ }^{15}$ H. A. Janssen, "Getreidedruck in Silozellen," Z. Oesterr. Ing.-Archit.-Ver. 39, 1045 (1895)

${ }^{16}$ J. Duran, Sands, Powders, and Grains: An Introduction to the Physics of Granular Materials (Springer, New York, 2000).

${ }^{17}$ B. Thomas, M. O. Mason, Y. A. Liu, and A. M. Squires, "Identifying states in shallow vibrated beds," Powder Technol. 57, 267 (1989).

${ }^{18}$ A. Ugawa and O. Sano, "Undulations of a thin granular layer induced by vertical vibration,” J. Phys. Soc. Jpn. 72, 1390 (2003).

${ }^{19}$ K. Kanai, A. Ugawa, and O. Sano, "Experiment on vibration-induced pattern formation of a vertically thin granular layer," J. Phys. Soc. Jpn. 74, 1457 (2005)

${ }^{20}$ C. R. Wassgren, C. E. Brennen, and M. L. Hunt, "Vertical vibration of a deep bed of granular material in a container," J. Appl. Mech. 63, 712 (1996).

${ }^{21}$ S. S. Hsiau and S. J. Pan, "Motion state transitions in a vibrated granular bed," Powder Technol. 96, 219 (1998).

${ }^{22}$ P. Eshuis, K. van der Weele, D. van der Meer, and D. Lohse, "The granular 
Leidenfrost effect," in Powders and Grains (Balkema, Leiden, 2005), Vol. 2, pp. 1155-1158.

${ }^{23}$ In a related 2-D numerical study, which showed a granular Leidenfrost effect with a strongly crystalline cluster, the aspect ratio was even smaller than 1. See Fig. 1 in B. Meerson, T. Pöschel, and Y. Bromberg, "Closepacked floating clusters: Granular hydrodynamics beyond the freezing point?" Phys. Rev. Lett. 91, 024301 (2003).

${ }^{24}$ E. Bodenschatz, W. Pesch, and G. Ahlers, "Recent developments in Rayleigh-Bénard convection," Annu. Rev. Fluid Mech. 32, 709 (2000).

${ }^{25}$ E. Clément and J. Rajchenbach, "Fluidization of a bidimensional powder," Europhys. Lett. 16, 133 (1991).

${ }^{26}$ J. B. Knight, H. M. Jaeger, and S. R. Nagel, "Vibration-induced size separation in granular media: The convection connection," Phys. Rev. Lett. 70, 3728 (1993).

${ }^{27}$ E. E. Ehrichs, H. M. Jaeger, G. S. Karczmar, J. B. Knight, V. Y. Kuperman, and S. R. Nagel, "Granular convection observed by magnetic resonance imaging," Science 267, 1632 (1995).

${ }^{28}$ K. M. Aoki, T. Akiyama, Y. Maki, and T. Watanabe, "Convective roll patterns in vertically vibrated beds of granules," Phys. Rev. E 54, 874 (1996).

${ }^{29}$ J. B. Knight, E. E. Ehrichs, V. Y. Kuperman, J. K. Flint, H. M. Jaeger, and S. R. Nagel, "Experimental study of granular convection," Phys. Rev. E 54, 5726 (1996)

${ }^{30}$ S. S. Hsiau and C. H. Chen, "Granular convection cells in a vertical shaker," Powder Technol. 111, 210 (2000).

${ }^{31}$ R. D. Wildman, J. M. Huntley, and D. J. Parker, "Convection in highly fluidized three-dimensional granular beds," Phys. Rev. Lett. 863304 (2001).

${ }^{32}$ A. Garcimartin, D. Maza, J. L. Ilquimiche, and I. Zuriguel, "Convective motion in a vibrated granular layer," Phys. Rev. E 65, 031303 (2002).

${ }^{33}$ S. S. Hsiau, P. C. Wang, and C. H. Tai, "Convection cells and segregation in a vibrated granular bed," AIChE J. 48, 1430 (2002).

${ }^{34} \mathrm{C}$. H. Tai and S. S. Hsiau, "Dynamics behaviors of powders in a vibrating bed," Powder Technol. 139, 221 (2004).

${ }^{35}$ G. M. Rodriguez-Linan and Y. Nahmad-Molinari, "Granular convection driven by shearing inertial forces," Phys. Rev. E 73, 011302 (2006).

${ }^{36}$ J. A. C. Gallas, H. J. Herrmann, and S. Sokolowski, "Convection cells in vibrating granular media," Phys. Rev. Lett. 69, 1371 (1992).

${ }^{37}$ Y.-h. Taguchi, "New origin of a convective motion: Elastically induced convection in granular materials," Phys. Rev. Lett. 69, 1367 (1992).

${ }^{38}$ S. Luding, E. Clément, A. Blumen, J. Rajchenbach, and J. Duran, "The onset of convection in molecular dynamics simulations of grains," Phys. Rev. E 50, R1762 (1994).

${ }^{39}$ M. Bourzutschky and J. Miller, "Granular convection in a vibrated fluid," Phys. Rev. Lett. 742216 (1995).

${ }^{40}$ Y. Lan and A. D. Rosato, "Convection related phenomena in granular dynamics simulations of vibrated beds," Phys. Fluids 9, 3615 (1997).

${ }^{41}$ K. M. Aoki and T. Akiyama, "Control parameter in granular convection," Phys. Rev. E 584629 (1998).

${ }^{42}$ R. Ramírez, D. Risso, and P. Cordero, "Thermal convection in fluidized granular systems," Phys. Rev. Lett. 85, 1230 (2000).

${ }^{43} \mathrm{P}$. Sunthar and V. Kumaran, "Characterization of the stationary states of a dilute vibrofluidized granular bed," Phys. Rev. E 64, 041303 (2001).

${ }^{44}$ J. Talbot and P. Viot, "Wall-enhanced convection in vibrofluidized granular systems," Phys. Rev. Lett. 89, 064301 (2002).

${ }^{45}$ P. Cordero, R. Ramirez, and D. Risso, "Buoyancy driven convection and hysteresis in granular gases: Numerical solution," Physica A 327, 82 (2003).

${ }^{46}$ D. Risso, R. Soto, S. Godoy, and P. Cordero, "Friction and convection in a vertically vibrated granular system," Phys. Rev. E 72, 011305 (2005).

${ }^{47}$ H. Hayakawa, S. Yue, and D. C. Hong, "Hydrodynamic description of granular convection," Phys. Rev. Lett. 75, 2328 (1995).

${ }^{48}$ X. He, B. Meerson, and G. Doolen, "Hydrodynamics of thermal granular convection," Phys. Rev. E 65, 030301 (2002).

${ }^{49} \mathrm{~T}$. Ohtsuki and T. Ohsawa, "Hydrodynamics for convection in vibrating beds of cohesionless granular materials," J. Phys. Soc. Jpn. 721963 (2003).

${ }^{50}$ E. Khain and B. Meerson, "Onset of thermal convection in a horizontal layer of granular gas," Phys. Rev. E 67, 021306 (2003).

${ }^{51}$ G. Miao, K. Huang, Y. Yun, and R. Wei, "Active thermal convection in vibrofluidized granular systems," Eur. Phys. J. B 40, 301 (2004).

${ }^{52}$ I. Goldhirsch, "Rapid granular flows," Annu. Rev. Fluid Mech. 35, 267 (2003).

${ }^{53}$ J. Eggers, "Sand as Maxwell's demon," Phys. Rev. Lett. 83, 5322 (1999).

${ }^{54}$ To keep the phase diagram well proportioned, not all data points of the Leidenfrost-convection transition (+) are presented in Fig. 14. The full set of data points can be seen in Fig. 11.

${ }^{55}$ S. B. Savage, "Streaming motions in a bed of vibrationally fluidized dry granular material," J. Fluid Mech. 194, 457 (1988).

${ }^{56}$ F. Melo, P. B. Umbanhowar, and H. L. Swinney, "Hexagons, kinks, and disorder in oscillated granular layers," Phys. Rev. Lett. 75, 3838 (1995).

${ }^{57}$ T. H. Metcalf, J. B. Knight, and H. M. Jaeger, "Standing wave patterns in shallow beds of vibrated granular material," Physica A 236, 202 (1997).

${ }^{58}$ C. Bizon, M. D. Shattuck, J. B. Swift, W. D. McCormick, and H. L. Swinney, "Patterns in 3D vertically oscillated granular layers: Simulation and experiment," Phys. Rev. Lett. 80, 57 (1998).

${ }^{59}$ N. Mujica and F. Melo, "Solid-liquid transition and hydrodynamic surface waves in vibrated granular layers," Phys. Rev. Lett. 805121 (1998).

${ }^{60} \mathrm{~N}$. Mujica, L. Caballero, and F. Melo, "Collective motion and solid-liquid type transitions in vibrated granular layers," Physica A 263, 362 (1999).

${ }^{61} \mathrm{O}$. Sano, "Random motion of a marker particle on square cells formed on vertically vibrated granular layers," J. Phys. Soc. Jpn. 681769 (1999).

${ }^{62}$ P. B. Umbanhowar and H. L. Swinney, "Wavelength scaling and square/ stripe and grain mobility transitions in vertically oscillated granular layers," Physica A 288, 344 (2000).

${ }^{63}$ D. Blair, I. S. Aranson, G. W. Crabtree, V. Vinokur, L. S. Tsimring, and C. Josserand, "Patterns in thin vibrated granular layers: Interfaces, hexagons, and superoscillons," Phys. Rev. E 61, 5600 (2000).

${ }^{64}$ J. M. Hill, M. J. Jennings, D. V. To, and K. A. Williams, "Dynamics of an elastic ball bouncing on an oscillating plane and the oscillon," Appl. Math. Model. 24, 715 (2000).

${ }^{65}$ J. R. de Bruyn, B. C. Lewis, M. D. Shattuck, and H. L. Swinney, "Spiral patterns in oscillated granular layers," Phys. Rev. E 63, 041305 (2001).

${ }^{66} \mathrm{~K}$. Kim and H. K. Pak, "Coarsening dynamics of striped patterns in thin granular layers under vertical vibration," Phys. Rev. Lett. 88, 204303 (2002).

${ }^{67}$ D. I. Goldman, J. B. Swift, and H. L. Swinney, "Noise, coherent fluctuations, and the onset of order in an oscillated granular fluid," Phys. Rev. Lett. 92, 174302 (2004)

${ }^{68}$ Y. S. Wong, C. H. Gan, C. H. Wang, X. Fan, D. J. Parker, A. Ingram, and J. P. K. Seville, "Instabilities in vertically vibrated granular beds at the single particle scale," Phys. Fluids 18, 043302 (2006).

${ }^{69}$ L. S. Tsimring and I. S. Aranson, "Localized and cellular patterns in a vibrated granular layer," Phys. Rev. Lett. 79, 213 (1997).

${ }^{70}$ T. Shinbrot, "Competition between randomizing impacts and inelastic collisions in granular pattern formation," Nature (London) 389, 574 (1997).

${ }^{71}$ S. J. Moon, J. B. Swift, and H. L. Swinney, "Role of friction in pattern formation in oscillated granular layers," Phys. Rev. E 69, 031301 (2004).

${ }^{72}$ J. Bougie, J. Kreft, J. B. Swift, and H. L. Swinney, "Onset of patterns in an oscillated granular layer: Continuum and molecular dynamics simulations," Phys. Rev. E 71, 021301 (2005).

${ }^{73}$ I. S. Aranson, L. S. Tsimring, and V. M. Vinokur, "Hexagons and interfaces in a vibrated granular layer," Phys. Rev. E 59, R1327 (1999).

${ }^{74}$ H. K. Park and H. T. Moon, "Square to stripe transition and superlattice patterns in vertically oscillated granular layers," Phys. Rev. E 65, 051310 (2002).

${ }^{75}$ H. M. Jaeger and S. R. Nagel, "Physics of the granular state," Science 255, 1523 (1992).

${ }^{76}$ H. M. Jaeger, S. R. Nagel, and R. P. Behringer, "Granular solids, liquids, and gases," Rev. Mod. Phys. 68, 1259 (1996).

${ }^{77}$ A. J. Liu and S. R. Nagel, "Jamming is not just cool any more," Nature (London) 396, 21 (1998).

${ }^{78}$ L. P. Kadanoff, "Built upon sand: Theoretical ideas inspired by granular flows," Rev. Mod. Phys. 71, 435 (1999).

${ }^{79}$ I. S. Aranson and L. S. Tsimring, "Patterns and collective behavior in granular media: Theoretical concepts," Rev. Mod. Phys. 78, 641 (2006). 